\title{
Heat transfer at in-line tube bank under low-frequency asymmetrical impulses impact on fluid flow
}

\author{
Aigul Khaibullina, Aidar Khairullin, Alexey Sinyavin, and Vladimir Ilin \\ "Energy supply of manufactures and energy resources saving technology" department, Kazan State \\ Power Engineering University, 420066 Kazan, Russia
}

\begin{abstract}
External heat transfer coefficient changing from corridor tube bundle to the reverse cross fluid flow with low-frequency asymmetrical pulsations was analyzed experimentally. The experiments were performed for Reynolds numbers in the tube bundle in the range of $100 \leq \mathrm{Re} \leq 500$ and changing of flow impulses frequency was limited with values $0.125 \leq \mathrm{f} \leq 0.5 \mathrm{~Hz}$, fluid pulsations amplitude in the tube bundle corresponded to $0.5 \mathrm{~d} \leq \mathrm{A} \leq 1.2 \mathrm{~d} \mathrm{~mm}$. A comparison of steady and non-steady conditions was made. The most efficient regime of pulsations in the studied range was found.
\end{abstract}

\section{Introduction}

The problem of heat transfer intensification is a topical problem for the heat power equipment. Different methods of heat exchange intensification are applied today.

By A. Bergls [1] was made the classification of sixteen different ways of heat transfer intensification, which were divided on active and passive methods. The most significant distinction between them is that the heat exchange intensification by passive methods doesn't need in external energy supply.

The problem of heat and mass transfer intensification has been studied comprehensively by the authors of [1].

The information about heat transfer intensification in the tube banks with heat exchange through the walls under condition of fluid flow pulsations is given in [2]. Also in [3] the results are reported about heat transfer intensification in the tube under the high-amplitude fluid flow vibrations which create a reverse flow.

In the article [4] authors point on heat exchange rate increase on average by $30 \%$ due to heat transfer agent pulsations in tube when Reynolds number is 1643 and impulses frequency is $1 \mathrm{~Hz}$. The date are given for fluid flows with Reynolds numbers in the range from 750 to 12320 , frequency range between 1 and $10 \mathrm{~Hz}$ and for different tubes diameters. The significant tube diameter influence on the heat transfer intensity has been shown.

Interesting results were obtained in investigation of low-frequency impulse impact on fluid flow to improve heat transfer conditions in laminar cross-flow around the solid body [5, 6].

The heat transfer in high frequency pulsing medium flowing in tube banks was also investigated in [7].

This is an Open Access article distributed under the terms of the Creative Commons Attribution License 4.0, which permits unrestricted use, distribution, and reproduction in any medium, provided the original work is properly cited. 


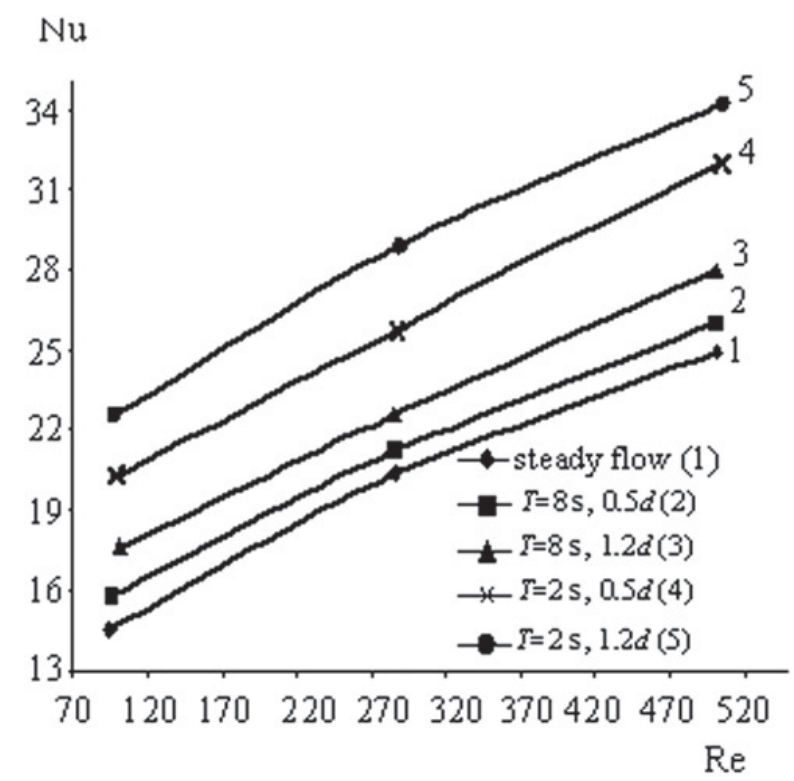

Figure 1. Dependence of Nusselt number changing from the Reynolds number with pulsations period $T=[2 ; 8] \mathrm{s}$ $(d-$ tubes diameter).

On the basis of chemical technology processes realization methods analysis was detected that pulsating form of processes realization is more effective in comparison with tradition approaches. The classifications of impact types were made by authors [8].

A literature review shows that the most of investigations directed on unsteady heat transfer are making abroad and high-frequency symmetrical pulsating flows are studied most often. The range of frequencies of asymmetrical fluid flow impulses lower than $1 \mathrm{~Hz}$ was not investigated comprehensively at flowing in tube banks.

\section{Experimental part}

The aim of the present work is impact analysis of low-frequency asymmetrical impulses applied to fluid flow on heat transfer. The characteristics are used: Reynolds numbers $\operatorname{Re}=[100 ; 500]$ and frequency $f=[0.125 ; 0.5] \mathrm{Hz}$.

Short review of investigation results are shown in Fig. 1.

The investigation results showed the intensification of heat transfer process by pulsations of fluid flow in comparison with heat exchange in steady flow on approximately $17 \%$. Figure 1 demonstrates that the greatest effect was obtained for the regimes with frequency $f=0.5$ and the amplitude of impulses $1.2 d$ (without taking into account the energy expenditures). It is need to be noted that increasing of the amplitude leads to heat transfer coefficient enlargement.

\section{Conclusions}

According to the research results can be made a conclusion that research of the impulses impact on heat transfer in the range of frequencies $f=[0.125 ; 0.5]$ and changing of amplitude can be potentially productive 


\section{References}

[1] Popov I.A., Mahyanov H.M., Gureev V.M. Physical basis and industrial application of heat transfer intensification: Heat transfer intensification: monograph / under the general editorship Uy.F. Gortishov. - Kazan: Innovative technologies center, (2009) -560 p

[2] Inaba, T., Kubo.T. Enhanced heat transfer through oscillatory flow // J. Heat Transfer - Japanese Research. (1993). Vol. 22. No. 5. P. 480-92

[3] Zhixiong Guo, Hyung Jin Sung. Analysis of the Nusselt number in pulsating pipe flow International Journal of Heat and Mass Transfer. (1997). Vol. 40. No. 10. P. 2486-9

[4] Zohir A.E., Habib M.A., Attya A.M., Eid A.I. An experimental investigation of heat transfer to pulsating pipe air flow with different amplitudes Heat and Mass Transfer. May (2006). Vol. 42. No. 7. P. $625-35$

[5] Olayiwola B.O., Walzel P. Flow pulsation and modified duct surface for process heat transfer intensification International Journal of Chemical Reactor Engineering. (2007). Vol. 5. P. 10

[6] Olayiwola B., Walzel P. Cross-flow transport and heat transfer enhancement in laminar pulsed flow // J. Chemical Engineering \& Processing: Process Intensification. (2008). Vol. 47. No. 5

[7] Bokun I.A. Heat exchange between a pulsating layer and a bundle of tubes immersed in it Vestsi Akademii Navuk BSSR, Serya Fizika Energetychnykh Navuk. (1977). No. 2. P. 37-40

[8] Promtov M.A. Machines and apparatuses with impulse power impact on in-process materials: Education guidance. - Moscow: "Machine engineering publishing - 1", (2004). - 136 p.

[9] V.K. Koshkin, E.K. Kalinin, G.A. Dreizer, S.A. Yarho. Unsteady heat transfer. Moscow, "Machine engineering”, (1973). -328 p. 